\title{
L'influence du modèle napoléonien en Espagne
} (1814-1845)

Jean-Philippe Luis

\section{(2) OpenEdition \\ 12 Journals}

\section{Édition électronique}

URL : https://journals.openedition.org/ahrf/1732

DOI : 10.4000/ahrf.1732

ISSN : 1952-403X

Éditeur :

Armand Colin, Société des études robespierristes

\section{Édition imprimée}

Date de publication : 1 juin 2004

Pagination : 199-219

ISSN : 0003-4436

\section{Référence électronique}

Jean-Philippe Luis, «L'influence du modèle napoléonien en Espagne (1814-1845) », Annales historiques de la Révolution française [En ligne], 336 | avril-juin 2004, mis en ligne le 15 juillet 2007, consulté le 23 avril 2022. URL : http://journals.openedition.org/ahrf/1732 ; DOI : https://doi.org/10.4000/ahrf.1732

Ce document a été généré automatiquement le 23 avril 2022.

Tous droits réservés 


\title{
L'influence du modèle napoléonien en Espagne (1814-1845)
}

\author{
Jean-Philippe Luis
}

1 Évoquer l'influence de l'expérience napoléonienne durant les décennies qui suivent la Guerre d'Indépendance espagnole peut sembler paradoxal pour un pays qui a massivement rejeté la tutelle de la France napoléonienne et a largement contribué à la chute de l'Empire. Napoléon a lui-même peu fait pour être perçu comme un inspirateur. Méprisant et méconnaissant l'Espagne, il a davantage cherché à imposer qu'à influencer. Sa vision de la Péninsule ibérique s'apparentait à celle d'une grande partie des élites françaises de son temps, formées avant la Révolution française à travers les clichés qui ont forgé la «légende noire de l'Espagne $»^{1}$. La connaissance de la cour de Charles IV qu'il avait acquise par les services diplomatiques n'avait fait que renforcer son mépris et son ignorance d'un pays pour lequel il n'a jamais eu l'attachement sentimental qu'il manifesta pour l'Italie.

2 L'influence de l'expérience napoléonienne en Espagne ne se manifeste cependant pas uniquement par un rejet total doublé de francophobie. Pour cerner cette influence, il convient de ne pas se limiter à la portée de ce que les Français firent entre 1808 et 1813 dans la Péninsule ibérique, mais d'étendre la réflexion dans l'espace et dans le temps. Par la déportation et par l'exil'2, des milliers d'Espagnols ont connu de l'intérieur la France napoléonienne puis celle de la Restauration et de la Monarchie de Juillet, comprenant par là comment le Consulat et l'Empire avaient transformé durablement le pays, au-delà de la chute du régime impérial. Cette connaissance de la France postnapoléonienne passait aussi en Espagne par les très nombreuses traductions d'ouvrages politiques, économiques et juridiques français. De ce fait, évoquer un modèle napoléonien est devenu pour une part importante des élites espagnoles évoquer un modèle d'État, de transformation économique et sociale qu'ils percevaient comme respectueux de l'ordre et comme ferment de la prospérité, un modèle alternatif au modèle anglais. Or, dans une Espagne en recul, le recours à des exemples étrangers était une nécessité : "l'Angleterre et la France marchent à la tête de la civilisation " ${ }^{3}$ écrivait Javier de Burgos au roi Ferdinand VII. Par ailleurs, la réception du modèle 
napoléonien en Espagne, fut largement conditionnée par la pensée et les expériences politiques (celle du despotisme éclairé, l'épreuve de la Guerre d'Indépendance et la longue lutte menée jusqu'aux années 1840 pour renverser définitivement l'Ancien Régime) des milieux susceptibles de s'y intéresser: ceux issus de la nébuleuse réformiste des ilustrados. Établir une différence entre modèle napoléonien et modèle bonapartiste n'est guère pertinent dans le cas espagnol. Les contemporains n'y faisaient d'ailleurs guère allusion, car ce qui intéresse avant tout dans l'expérience française est un exemple de réforme de l'État et de la société susceptible d'inspirer l'Espagne, mais tout en restant dans un strict cadre monarchique.

3 Seule cette perspective large permet de comprendre comment au rejet massif qui marque les années immédiatement postérieures à la Guerre d'Indépendance, succède une vision beaucoup plus nuancée, voire presque positive, à mesure que s'affirme en Espagne un libéralisme modéré, conservateur et autoritaire, principal promoteur d'un État libéral qui acquiert sa cohérence dans les années 1840.

De l'enfer au purgatoire : la mémoire de Napoléon et du règne de Joseph Bonaparte

4 L'image de Napoléon en Espagne n'est pas aussi monolithique que pourrait le laisser supposer l'intensité de la lutte des Espagnols contre les Français durant la Guerre d'Indépendance. En effet, l'établissement du Consulat fut plutôt bien accueilli, puis les opinions devinrent plus contrastées sur les débuts de l'Empire4. À partir de 1808, le rejet est sans nuance, à l'exception bien sûr des afrancesados, mais les motifs de ce rejet diffèrent. Pour les contre-révolutionnaires, Napoléon est l'incarnation du mal, "l'homme qui viole toutes les lois divines et humaines ". Les libéraux insistent plutôt sur son machiavélisme, son despotisme en minorant le génie militaire de l'empereur et la portée de ses mesures réformistes ${ }^{6}$.

5 Dans les années qui suivent la Guerre d'Indépendance, les voix discordantes sont rarissimes. La plus connue est probablement celle de Francisco Mina (pourtant neveu du général Ezpoz y Mina, un héros de la Guerre d'Indépendance) qui, en exil aux ÉtatsUnis, rendit hommage à Joseph Bonaparte ${ }^{7}$. Il faut attendre le Trienio liberal (1820-1823) pour voir se diffuser des images plus nuancées de l'Empire. À la curiosité à l'égard de la traduction des œuvres de Napoléon s'ajoutent les premiers signes de l'apparition de la légende napoléonienne qui assimile la gloire impériale au combat pour l'émancipation des peuples et pour la liberté8. La présence d'anciens soldats de l'Empire (en particulier le général Cugnet de Montarlot), engagés en Catalogne pour défendre le régime libéral espagnol contre l'invasion française des Cent mille fils de Saint Louis marque une collaboration limitée et conflictuelle, mais bien réelle, entre des libéraux européens (français et italiens pour l'essentiel) et les libéraux espagnols. Des cris « Vive Napoléon II » auraient été entendus à Barcelone ${ }^{9}$ et même à Madrid ${ }^{10}$. Ces faits restent cependant très limités et il faut attendre les années 1830-1840 pour trouver quelques vertus à l'empereur. On valorise alors chez une partie des libéraux modérés celui qui «coupa toutes les têtes de l'hydre révolutionnaire, rétablit la paix et l'ordre public ${ }^{11}$.

Cette évolution de l'image de Napoléon et de l'action du roi Joseph affecte aussi ceux qui ont collaboré avec l'occupant français. Le drame des afrancesados jusqu'au Trienio liberal a suscité depuis longtemps des travaux qui font toujours référence ${ }^{12}$. Le décret du 30 mai 1814, signé par Ferdinand VII quelques jours après la réussite de son coup d'État, organise une proscription à vie pour tous les anciens responsables de l'État joséphin et un placement en résidence surveillée pour leurs subalternes ${ }^{13}$. Enfin, les biens des 
exilés sont saisis. Ce texte organise non seulement la proscription, mais aussi une forme de déchéance civile pour ceux qui choisirent de rester en Espagne.

7 Après une première amnistie limitée en 1818 , le retour des libéraux au pouvoir en 1820 aurait pu marquer le pardon pour les afrancesados. Les décrets des Cortès du 23 avril et du 26 septembre 1820 organisent certes une véritable amnistie, mais la marque d'infamie qui les touche n'est pas effacée : les afrancesados ne retrouvent pas les emplois qu'ils occupaient avant 1808 et ne peuvent être ni éligibles ni électeurs aux Cortès ${ }^{14}$. Ils restent à certains égards des parias dans leur pays, sont attaqués dans la presse libérale comme dans la presse conservatrice ${ }^{15}$ et sont souvent mis à l'écart dans les nominations aux emplois publics ${ }^{16}$.

8 La déception à l'égard des libéraux qui fut la leur explique très largement le rapprochement qui s'est opéré avec les absolutistes modérés dès la chute du Trienio libera ${ }^{17}$. Pourtant, il n'y eut légalement aucune avancée, bien au contraire et certains durent reprendre le chemin de l'exil en 1823. L'amnistie dont ils avaient bénéficié (en 1820) était abolie comme toutes les lois libérales. Malgré la forte pression du duc d'Angoulême puis de la diplomatie française, les afrancesados continuent à être collectivement exclus de la communauté nationale ${ }^{18}$. Consulté à leur propos, le conseil de Castille affirme (le 30 octobre 1824) que l'oubli est impossible car « la part qu'eurent les afrancesados dans les désastres et les troubles (de la Guerre d'Indépendance) restent dans la mémoire des Espagnols fidèles ${ }^{19}$. Des retours ont pourtant lieu à la faveur des divisions de plus en plus profondes du camp absolutiste en modérés et ultras. Les modérés recherchent l'appui d'hommes d'expérience, bien introduits auprès de la place de Paris, pour négocier les emprunts indispensables à un Trésor Royal aux abois. Sans jamais occuper des emplois de premier plan, mais plutôt des commissions temporaires, quelques dizaines d'afrancesados (Javier de Burgos, Alberto Lista, Pedro Sainz de Andino, Sebastián de Miñano pour les plus connus) réapparaissent au sein de l'appareil d'État, ce qui déclenche la fureur des absolutistes ultras.

9 Les afrancesados ne cessent véritablement d'être exclus collectivement du corps social qu'à la faveur de l'épilogue troublé et chaotique du règne de Ferdinand VII. L'amnistie du 22 mars 1833 est totale et restitue aux personnes qu'elle affecte tous «leurs biens, décorations, honneurs et distinctions $»^{20}$. Dans les années qui suivent, la progressive construction d'un État libéral modéré contribue à réévaluer leur action et dans certains milieux à les réintégrer dans l'histoire nationale. L'article afrancesados d'un dictionnaire de la politique publié en 1849 expliquait avec indulgence : «il y eut des hommes qui aimaient tant la liberté qu'ils l'ont préférée, même imposée par les étrangers, au despotisme national $»^{21}$.

10 Cette évolution ne traduit pas uniquement l'œuvre du temps qui aurait apaisé les passions. Elle est liée, chez ceux qui rejetaient dans un même temps Ancien Régime et dérive démocratique, à la prise de conscience de l'intérêt du modèle napoléonien pour l'Espagne. Cela s'est traduit principalement par l'adoption de réformes administratives et juridiques qui, au premier abord, semblent s'inspirer largement du modèle français. Le modèle de l'État napoléonien et l'EspagneUne influence majeure mais tardive

11 On connaît l'ambition napoléonienne de procéder à une révolution européenne du droit, clef de voûte d'une confédération dominée par la France. Si cet effort produisit des effets immédiats et durables en Italie et dans les pays rhénans, moyennant une adaptation aux sociétés concernées ${ }^{22}$, il faut attendre en Espagne la fin des années 1830 pour qu'émerge un modèle durable d'État libéral, sous l'impulsion des libéraux 
modérés, un État dont les fondements et les structures semblent présenter certaines similitudes avec le modèle napoléonien. Ce décalage chronologique est lié à deux facteurs : le poids de la Guerre d'Indépendance et la concurrence du modèle anglais.

a. La Guerre d'Indépendance ruina pour un temps la portée de l'adoption des codes uniformisateurs et des réformes administratives entamées sous Joseph Bonaparte. Pendant de nombreuses années, il devient impossible de revendiquer une inspiration française. Pourtant, la ilustración représentait un terreau particulièrement favorable à un intérêt pour l'œuvre administrative et juridique de Bonaparte. D'ailleurs, une bonne partie des mesures contenues dans la Constitution de Bayonne, dans les décrets de Chamartín ou dans les lois adoptées par le roi Joseph, étaient celles qu'appelaient de leurs vœux une grande partie des réformateurs avant 1808 (vente des biens du clergé, refonte de l'appareil d'État, suppression des Conseils, adoption de codes unifiant le pays...) et que reprirent en grande partie les libéraux. Les hésitations dans l'établissement d'un ministère de l'Intérieur représentent un bon exemple de la difficulté à solder les comptes de la Guerre d'Indépendance ${ }^{23}$. Le décret du 6 février 1809 adopté par Joseph Bonaparte instaurait un ministère de l'Intérieur chargé de l'administration civile du royaume et un ministère de la " Police générale ». Au même moment, les libéraux de Cadix préféraient regrouper les attributions de ces ministères dans un secrétariat d'État de la Gobernación. L'absolutisme restauré par deux fois supprime ces ministères, mais durant la dernière décennie de l'Ancien Régime (1823-1833), la réforme administrative est progressivement perçue comme la seule voie politiquement acceptable pour sauver l'absolutisme. De ce fait, la nécessité de ministères s'occupant exclusivement de l'administration civile et de la police s'impose car ces fonctions étaient éparpillées entre les différents secrétariats d'État, les Conseils ainsi que les audiences et chancelleries qui siégeaient sur tout le territoire. Les réformateurs de l'absolutisme font pression sur Ferdinand VII pour imposer la réforme, aidés en cela par quelques afrancesados. La tâche était cependant très délicate car il fallait absolument éviter tout parallèle avec l'expérience française. Le marquis de la Almenara, un afrancesado, prévenait dans un projet de réforme de 1826: "on ne propose pas de ministère de l'Intérieur car l'ignorance a fait dire que ce ministère avait été le bras droit de la Révolution Française ${ }^{24}$. Les absolutistes ultras font échouer toutes les tentatives jusqu'en 1832, date à laquelle la rupture est consommée à l'intérieur du camp absolutiste. Apparaît alors un nouveau ministère, qui ne s'appelle pas ministère de l'Intérieur (appellation trop afrancesada), ni de la gobernación (appellation trop libérale), mais ministère du Fomento, reprenant un terme issu de la tradition du réformisme absolutiste d'avant 1808.

b. Le modèle anglais a été pendant longtemps un concurrent du modèle français. En effet, avant 1808, l'esprit anglais imprégnait déjà fortement la ilustración espagnole ${ }^{25}$. L'aide anglaise aux patriotes espagnols entre 1808 et 1814 a renforcé encore davantage cette influence. Tout en étant certes influencée par la Constitution française de 1791, la Constitution de Cadix de 1812 témoigne de l'inspiration anglaise dans l'indépendance absolue du pouvoir judiciaire, dans son monopole juridictionnel ${ }^{26}$ ainsi que dans le poids dominant de l'assemblée par rapport à l'Exécutif, en particulier dans la tutelle de l'appareil administratif. Si l'influence anglaise recule après 1823 c'est parce que le modèle constitutionnel élaboré à Cadix et repris entre 1820 et 1823 est perçu comme un échec. Le régime d'assemblée est rejeté car rendu responsable du désordre, de l'agitation révolutionnaire qui aurait entraîné la chute du régime libéral et la restauration de l'absolutisme en $1823^{27}$. Chez la majorité des libéraux, l'héritage du 
despotisme éclairé, rajeuni par l'exemple français de l'État napoléonien s'impose alors complètement et d'autant plus facilement qu'une partie des absolutistes se convertit durant la seconde restauration à la réforme et à la rationalisation du vieil appareil d'État d'Ancien Régime. Modérés du libéralisme et de l'absolutisme se retrouvent après la mort de Ferdinand VII en 1833 pour élaborer un modèle étatique libéral qui donne désormais la primeur à l'Exécutif dans un État organisé selon trois principes administratifs : unité, centralisation, hiérarchie ${ }^{28}$.

L'influence française dans les étapes de la construction d'un État libéral

En France, l'œuvre administrative et codificatrice de Bonaparte est le fruit d'une systématisation d'initiatives antérieures, celles des juristes rationalisateurs de la monarchie absolue des XVIIe et XVIIIe siècles, des hommes de Lumières et celles bien sûr de la Révolution française ${ }^{29}$. En Espagne, la ilustración, a bien sûr subi l'influence française au travers des philosophes, mais aussi au travers des travaux de juristes, en particulier ceux de Jean Domat, le précurseur des études administratives françaises. Son Les lois civiles dans leur ordre naturel; le droit public et legum delectus, qui connut plusieurs publications depuis 1696, fut traduit pour la première fois en Espagne en 1788. Domat est un des principaux inspirateurs du grand juriste catalan Ramón Lázaro de Dou y Bassols, auteur du monumental Derecho público interno o Instituciones de Derecho público general de España con referencia al particular de Cataluña, dont les 9 tomes sont publiés entre 1800 et $1804^{30}$. Cet homme, qui participa aux débats des Cortès de Cadix, représente un pont entre la mise en ordre des théories traditionnelles et l'avènement d'un véritable droit administratif dans les années 1830-1840.

Les libéraux de Cadix tout comme ceux du Trienio liberal n'ont pas élaboré un véritable modèle politico-administratif clair, faute de temps, mais aussi faute de choix entre un régime d'assemblée et une stricte séparation des pouvoirs. Après 1823 , le modèle français semble le plus efficace et le plus approprié à l'Espagne car il peut s'inscrire dans le prolongement de la tradition ilustrada. Deux étapes sont discernables. La première a lieu durant la deuxième restauration de Ferdinand VII, entre 1823 et 1833 et est fortement marquée par des hommes qui furent afrancesados. Ces derniers développent une véritable idéologie "administrative" qui voit dans la réforme de l'administration la solution à tous les maux dont souffre le pays ${ }^{31}$. À partir de 1826, cette poignée d'hommes exerce une influence non négligeable au secrétariat d'État aux Finances et au secrétariat d'État aux affaires d'État. Le plus célèbre d'entre eux, Javier de Burgos, ancien sous-préfet d'Almeria sous Joseph, voyait dans la centralisation et l'uniformisation le fruit des progrès de la science de l'administration, «science de ce qui est utile et nuisible», comme "le droit est la science de ce qui est juste et injuste $\aleph^{32}$. Burgos, comme tous les réformateurs, préconisait la séparation entre les activités administratives et judiciaires, ce qui signifiait la relégation des Conseils, des audiences et chancelleries au seul rang de tribunaux d'appel. Il réclamait encore la création d'un ministère de l'Intérieur chargé aussi du fomento, c'est-à-dire du développement économique du pays. Une fois ministre de Fomento en 1833, Burgos est le très célèbre promulgateur de la première division administrative uniforme du pays (décret du 30 novembre 1833) qui crée 49 provinces dont deux insulaires, dirigées par des représentants de l'État: subdelegados de fomento puis gobernadores civiles à partir du décret du 13 mai 1834. L'inspiration française est évidente dans cette réforme, avec bien sûr des nuances, en particulier car les gouverneurs civils n'auront jamais les mêmes pouvoirs que les préfets. L'initiative de Burgos est l'épilogue d'une série de projets de réforme territoriale qui ont débuté sous Joseph Bonaparte et se sont 
prolongés sous les régimes libéraux ainsi que sous la deuxième restauration de l'absolutisme ${ }^{33}$.

Durant cette dernière période, Pedro Sainz de Andino, l'autre afrancesado actif, ancien sous-préfet tout comme Burgos, réintègre discrètement le ministère des Finances en 1828 pour rédiger au sein d'une commission le premier code du commerce espagnol promulgué en 1829. L'un des cinq membres de cette commission, Manuel María Cambronero, était lui aussi afrancesado, ancien conseiller d'État sous Joseph Bonaparte $^{34}$. En 1831, Sainz de Andino présente enfin un projet de code criminel qui n'est cependant pas adopté.

L'avènement du régime libéral en 1834 permet de procéder aux réformes administratives que l'absolutisme finissant souhaitait mais ne pouvait se permettre politiquement de mener à bien : suppression des conseils, séparation du judiciaire et de l'administratif, création de véritables ministères, mise en place d'une administration provinciale sur un modèle uniforme. Cependant, la première guerre carliste, les difficultés financières qu'elle génère, l'opposition entre libéraux progressistes -plus sensibles à certains aspects du modèle anglais- et libéraux modérés, rendent la situation institutionnelle et juridique confuse. La stabilisation n'intervient vraiment qu'à partir des années 1840 avec la victoire durable des modérés. Ces derniers imposent un État qui, pour se légitimer, a largement recours à l'exemple d'outre Pyrénées et en particulier au droit administratif français. Ce dernier, né sous le Consulat ${ }^{35}$, connaît un grand succès auprès des Espagnols qui connurent l'exil en France dans les années 1820. À partir de 1834, ses principaux auteurs sont traduits en Espagne. Les Principes d'administration publique de Bonnin, publiés à Paris en 1808, sont traduits sous une forme abrégée en $1834^{36}$; L'Abrégé du droit administratif à l'usage des étudiants en droit, de Gandillot, publié à Paris en 1833 est traduit deux ans plus tard. Les juristes espagnols qui vantent ce modèle (Burgos, le précurseur, puis Silvela, Oliván ou Posada Herrera pour les plus connus) sont aussi des hommes politiques, liés au parti modéré et sont considérés comme les fondateurs du droit administratif espagnol. La progressive mise en place de ce nouvel État libéral s'accompagne comme nous l'avons vu plus haut, d'une relecture du passé dans un sens moins défavorable aux afrancesados.

L'influence française est telle en la matière qu'elle génère une polémique aux Cortès puis dans la presse en 1839-1840. En avril 1840, Lasagra, un député anglophile s'écriait : " quel droit a la France à s'ériger en matière administrative comme la première nation du monde et à nous imposer ses lois administratives? [...] N'y a-t-il pas d'autres étrangers que les Français?» Cette opinion n'était cependant pas dominante. L'adoption du modèle français se faisait soit sans états d'âme, soit en ayant recours à une argumentation utilitariste. Le ministre Francisco Agustín Silvela illustre le premier cas de figure en écrivant en 1839: «L'administration française nous offre un degré notable de perfection, si nous la comparons avec celles d'autres pays que nous connaissons aussi plus ou moins; par conséquent, nous devons y recourir $»^{37}$. De son côté, le député Alejandro Oliván rejetait les accusations de francophilie : «Ce n'est pas l'administration française que l'on recherche, sinon la bonne administration ${ }^{38}$. Enfin, se défendant de ne faire que copier la France, Burgos, Silvela ou Oliván se revendiquaient de l'héritage de Gaspar de Jovellanos ${ }^{39}$, faisant ainsi le lien avec la tradition ilustrada.

L'adoption d'un tel modèle a pour conséquence la mise en place d'une administration toute puissante. En France, la Constitution de l'an VIII avait vu «l'absorption de toute 
l'administration dans la sphère gouvernementale ». Désormais, « seul le gouvernement définit le régime juridique interne des services publics » et « détermine les règles entre individus et autorités administratives $»^{40}$. Les mêmes phénomènes affectent l'État libéral espagnol quarante ans plus tard. Une série de mesures prises de 1839 à 1845 permettent à l'administration d'échapper totalement au pouvoir législatif et au pouvoir judiciaire : le droit de juger l'administration doit venir de l'administration elle-même et non des juges. Le processus s'achève en 1845 avec la création d'une juridiction autonome du contentieux administratif ${ }^{41}$. Cette mesure correspond cependant à une systématisation des différents privilèges juridictionnels dont bénéficiaient les employés de l'État sous l'Ancien Régime. Cette origine ancienne que l'on retrouve dans la genèse de nombreuses innovations, ainsi que la revendication de la filiation de Jovellanos montrent comment l'influence française indéniable s'inscrit dans le prolongement d'une voie clairement espagnole : celle du réformisme autoritaire élaborée au XVIIIe siècle. Ainsi, dans la mesure où tout modèle juridico-administratif repose sur une idéologie ou une option politique, peut-on établir des liens idéologiques entre le bonapartisme et la nébuleuse espagnole des réformateurs modérés?

Idéologie bonapartiste, afrancesados et moderados

Tenter de cerner l'influence de l'idéologie bonapartiste en Espagne n'est pas chose facile car, comme l'a montré Antoine Casanova ${ }^{42}$, la pensée politique de l'empereur ne représente pas un ensemble cohérent. Les fluctuations dans les analyses et les contradictions ne manquent pas et le discours sert souvent à justifier a posteriori l'action. Par ailleurs, Joseph n'est pas Napoléon: le premier avait pour l'Espagne un projet plus respectueux des hiérarchies traditionnelles et de l'influence de l'Église. Joseph entendait s'appuyer sur les élites intellectuelles et administratives du pays et l'on ne retrouve pas en Espagne les accents jacobins, l'appel à la lutte contre les ordres privilégiés et pour la liberté, que la propagande bonapartiste a revêtu en Italie ou en Allemagne. Quelques relations fortes peuvent toutefois être dégagées.

L'afrancesamiento comme une des sources du modérantisme

Il est établi depuis l'œuvre pionnière de Miguel Artola que l'afrancesamiento n'est pas un mouvement importé, mais est issu du courant réformateur de la fin du XVIIIe siècle espagnol qui a pour projet de lever les blocages de la société d'Ancien Régime. C'est à partir de cette même matrice culturelle et intellectuelle que certains acceptèrent la dynastie française, d'autres, les libéraux, rejoignirent le camp des patriotes. De ce fait, le choix en faveur d'un camp ou d'un autre est davantage le fruit des circonstances, des relations familiales, des amitiés, de la proximité culturelle avec la France, de l'analyse des événements à chaud que d'un choix idéologique. On trouve ainsi parmi les afrancesados un large éventail d'opinions: de l'héritier du despotisme éclairé au républicain, en passant par le monarchiste libéral ${ }^{43}$. La majorité d'entre eux furent cependant effrayée par l'irruption brutale du peuple dans le combat politique. Ils en restent d'autant plus attachés aux conceptions dominantes qui prévalaient avant 1808 parmi les élites et chez les réformateurs: les changements profonds nécessaires doivent se faire sans intervention populaire, dans le respect de l'ordre social ${ }^{44}$. Les afrancesados n'eurent pas l'impression de trahir leur pays, mais de choisir une option plus efficace que celle prise par leurs amis patriotes libéraux pour réformer le royaume. Il est significatif que le cardinal Llorente se qualifie de "constitutionnel de l'année $1808 »$ et refuse la division avec les constitutionnels de Cadix ${ }^{45}$. 
Après la Guerre d'Indépendance et l'exil en France, les afrancesados ne représentent jamais un parti. Seuls quelques-uns d'entre eux, des hommes qui étaient jeunes et eurent des responsabilités de second plan durant la Guerre d'Indépendance, jouent à nouveau un rôle politique à partir du Trienio liberal. Ils se regroupent autour de journaux, El Sol puis El Censor, La miscelanea, et forment un cercle étroit dominé par Javier de Burgos, Felix Reinoso, Alberto Lista, Sebastián Miñano et José Gómez Hermosilla. Ils s'opposent alors aux libéraux exaltés, se rapprochent des libéraux modérés, mais restent cependant déçus par l'amnistie trop limitée dont ils ont bénéficié. Les exaltés au pouvoir à partir de juillet 1822 interdisent les journaux afrancesados, ce qui accentue la rupture avec le régime du Trienio liberal ${ }^{46}$. Cette opposition explique le rapprochement qui s'opère avec l'absolutisme de Ferdinand VII à partir du moment où celui-ci est contraint à la réforme. Comme je l'ai souligné plus haut, la plupart de ces hommes participent à des commissions chargées de l'élaboration des réformes et introduisent en Espagne les techniques administratives françaises. Lista obtient même l'autorisation de publier à partir de 1831 des journaux (La Gaceta de Bayona, La Estafeta de San Sebastián et La Estrella) vantant une politique de réforme qui ne remettrait pas en cause la légitimité et l'autorité royale ${ }^{47}$.

Après la mort de Ferdinand VII, le rapprochement idéologique avec les libéraux modérés se confirme, malgré les difficultés ${ }^{48}$, et est facilité par l'abandon par ces derniers du modèle de la Constitution de Cadix et par l'adoption durant l'exil des thèses du libéralisme doctrinaire et de l'utilitarisme de Bentham, dépouillé de ses conséquences politiques ultimes. Le résultat est la constitution de ce vaste courant modéré dont Lista énonçait déjà en 1809 deux principes fondamentaux dans $\mathrm{El}$ Espectador Sevillano : «l'équidistance » entre l'absolutisme ("pouvoir arbitraire») et la démocratie (" anarchie »); et l'application institutionnelle du juste milieu : l'équilibre des pouvoirs entre les Cortès et le Roi $»^{49}$.

La primeur à la " clase media »

Quelques idées force de l'idéologie napoléonienne se retrouvent dans la genèse du modérantisme. La première est la place centrale accordée à la classe moyenne dans le processus de modernisation du pays et dans la direction de celui-ci. Elle est « la base, le support, l'agent historique de ce développement du "ferment industriel" et de l'essor des idées, des Lumières $»^{50}$. "Elle est nécessaire pour encadrer politiquement les énergies populaires [...], précieuses et (si elles ne sont pas canalisées) redoutables ». "Sans cette classe moyenne aucune perspective révolutionnaire constructive ne peut être viable $\aleph^{51}$. Cette vision est partagée par les réformateurs espagnols de la fin du XVIIIe siècle qui voient dans ce groupe social « libéré des vices de l'aristocratie et de la plèbe, les principaux dépositaires de ces valeurs -austérité, travail, probité, solvabilitésur lesquelles doit s'appuyer la recherche du progrès matériel et la régénération morale et politique de la société $\aleph^{52}$. Cette opinion est centrale dans la pensée des afrancesados, mais elle s'accompagne d'une nuance dans les relations à établir avec le peuple. Napoléon estime la " classe véhémente et nombreuse, facile à soulever car elle est sans propriété et facile à conduire car elle est sans lumière $»^{53}$. Néanmoins, il vante son « désintéressement, le courage civique, l'attachement héroïque à la Révolution et à l'indépendance de la Nation » et son utilité dans le processus historique car il demeure persuadé que le changement politique, parce qu'il est porteur de changement social, ne peut qu'être violent et donc de nature révolutionnaire, d'où l'alliance nécessaire avec « les classes indigentes et laborieuses $»^{54}$. 

reconnaitre le sens du sacrifice que manifeste le peuple dans la lutte contre les Français. C'est précisément pour cette dernière raison que les afrancesados campent sur les positions qui étaient celles de la ilustración : la réforme pour être durable ne peut se faire qu'en l'absence de l'intervention populaire, dans l'ordre, dans le respect des hiérarchies sociales qui se modifieront naturellement à mesure que les réformes porteront leurs fruits. Sous le Trienio liberal, cette question de l'alliance avec le peuple marque le point de rupture entre libéraux exaltés et modérés, ces derniers ayant rejoint les positions des afrancesados. Par la suite, ceux-ci évoluent comme nous l'avons $\mathrm{vu}$, dans un sens encore plus conservateur, ce qui les conduit à revenir sur le rôle attribué à la clase media. Dans une lettre écrite en 1830 à son ami Reinoso, Lista affirmait : «la maladie commune aujourd'hui à toute l'Europe se réduit à ceci : la clase media veut gouverner, ce qui fut le péché originel de 1789 ». Dans une telle situation, deux cas de figure se présentent: soit «il se forme au sein de la clase media une aristocratie militaire qui amène le despotisme à des peuples fatigués de l'anarchie ", soit « le pouvoir descend de la clase media aux prolétaires, dont l'unique science de gouvernement se réduit au terrorisme $»^{55}$. De ce fait, une société est bien organisée quand « la classe dirigeante la dirige, la clase media l'enrichit de ses produits et de ses connaissances et la multitude lui prête ses forces physiques pour toutes les opérations nécessaires $\Perp^{56}$. Sans aller aussi loin, les moderados des années 1830-1840 s'avèrent beaucoup moins enthousiastes à l'égard de la vision centrale que doit jouer la classe moyenne et privilégient une solution politique et constitutionnelle qui limite la vie politique à une élite réduite. Les afrancesados furent les précurseurs d'une telle évolution qui, dès les années 1820 , ambitionne de faire émerger une société inerte politiquement, la politique se dissolvant dans «une action administrative efficace, capable d'assurer la sécurité des biens et des personnes et la multiplication des moyens de subsistance $\aleph^{57}$. La tradition ilustrada - afrancesada rejoint alors dans les années 1830 les théories doctrinaires françaises qui ont tendance à limiter «la politique à une simple gestion $»^{58}$. La conséquence en est la nécessaire réduction de l'espace dévolu au débat public et donc une limitation de la liberté de la presse et de réunion.

De l'ordre comme garant de la liberté à la limitation de la liberté politique

Tout en inventant l'opinion publique, les ilustrados souhaitaient sa limitation à la partie la plus éclairée de la nation, mais, dans le même temps, l'absolutisme réformateur découvrait l'intérêt de l'opinion et de la manipulation de cette dernière par l'utilisation contrôlée de la presse et des nouveaux lieux de débat. Avec l'héroïsation du peuple pendant la Guerre d'Indépendance, les afrancesados furent les seuls à camper sur ces positions. Ils sont ensuite rejoints par les libéraux modérés à partir de la fin de la Guerre d'Indépendance et surtout à partir du Trienio liberal ${ }^{59}$. La collaboration avec les absolutistes dans les années 1826-1834 durcit les positions des afrancesados. Lista affirmait en 1830 que les discussions de haute politique pouvaient être très utiles « en France, en Angleterre et dans d'autre pays ; en Espagne, tout ce qui produit des partis est mortifère $»^{60}$. Javier de Burgos, alors ministre de la Gobernación, promulgue le 4 janvier 1834 un décret qui maintient la censure préalable sur la presse et les livres à l'exception des textes strictement scientifiques. Avec le retour des libéraux, la censure se relâche, mais la limitation de la liberté de la presse est toujours pensée comme une nécessité par les modérés au pouvoir qui revendiquent la distinction célèbre de Benjamin Constant entre liberté civile et liberté politique. 

limiter le débat public, mais plutôt du fait qu'en Espagne, personne n'eut « le sens de la publicité $\aleph^{61}$ qui caractérisa très tôt le jeune Bonaparte. Même la propagande qui entoura Espartero, le vainqueur de la guerre carliste, durant sa régence (1840-1843) ne réussit pas de manière durable à combiner comme le fit Napoléon la gloire militaire, l'énergie constante et personnelle mise dans le travail de propagande, et la volonté de maintenir un lien privilégié avec la Nation que Napoléon était persuadé d'incarner.

Souveraineté et Nation modérée espagnole doit fondamentalement être reliée à la place accordée aux masses dans chacun des cas. En Espagne, ceux qui furent le plus influencés par la France de Napoléon ont toujours exclu totalement les masses de leurs schémas de pensée. La Guerre d'Indépendance qui a vu l'irruption brutale du peuple dans une dynamique contre-révolutionnaire y est probablement pour beaucoup, tout comme, mais dans une moindre mesure, les émeutes et les violences révolutionnaires du Trienio liberal. Tout en les excluant du jeu politique, Bonaparte reconnaissait, comme nous l'avons vu, leur poids historique et leur bravoure. Le peuple reste la "source sacrée de tous les pouvoirs" -ce qui est symbolisé par le suffrage universel- même "s'il n'en exerce aucun ». Ainsi, d'après Pierre Rosanvallon, Bonaparte «a renoué avec le mythe primitif de la monarchie élective en réinventant le despotisme éclairé », répondant ainsi à la tension entre "le nombre et la raison $»^{62}$. Dès la Guerre d'Indépendance, il n'y eut rien de tel en Espagne, ni dans l'expérience du roi Joseph, ni chez les afrancesados, ni a fortiori dans le conglomérat modéré qui domine à partir des années 1840. Dès l'origine, le mouvement réformateur est marqué à la fin du XVIIIe siècle par un discours historiciste de plus en plus prégnant pour justifier l'origine du pouvoir. Chez Jovellanos, la nation n'est pas l'association de citoyens libres et égaux mais une identité historique fondée sur les différents corps qui composent cette nation ${ }^{63}$. Avec les modérés, la souveraineté n'est pas le fruit de la volonté du corps social, mais un produit de l'histoire qui a vu l'apparition d'institutions durables, la plus importante d'entre elles étant la monarchie ${ }^{64}$. Cette perception rejoint la conception originale de la Nation qui s'est imposée avant même 1808 : une communauté supérieure d'origine historique à laquelle sont soumis les individus ${ }^{65}$.

afrancesados sont héritiers de cette pensée qui prend de plus en plus d'importance à mesure que recule le rationalisme universaliste des Lumières. À la fin du règne de Ferdinand VII, Lista va même jusqu'à reprendre l'argument conservateur de la légitimité de fait de tout gouvernement: "la création de tout gouvernement est l'œuvre des hommes, de l'expérience et des circonstances ; mais une fois la loi établie, celui-ci est légitimé, tombe sur lui la sanction divine, comme sur toutes les institutions conservatrices de la société ${ }^{66}$. Les modérés reprennent à partir des années 1830 l'argument historique pour rejeter le principe de la souveraineté nationale, le pouvoir étant un attribut de la raison, et non pas de la volonté humaine, la souveraineté réside dans la monarchie ${ }^{67}$. Dans cette perspective, les individus, comme l'écrivait en 1843-1844 un Alcalá Galiano devenu modéré, n'ont en somme qu'un seul droit véritable : «celui d'être gouverné bien et avec justice ${ }^{68}$.

Économie et politique

La place centrale de l'économie et des transformations sociales que son évolution génère est l'autre grand point commun entre le bonapartisme et la nébuleuse 
réformiste modérée espagnole. Héritier de la pensée des Lumières, Bonaparte est un farouche partisan de la levée des entraves que la société d'Ancien Régime faisait peser sur le développement économique. La Révolution s'enracine pour lui dans «le ferment industriel », mais la lecture de Rousseau et sa propre expérience politique lui font percevoir le changement technique comme le catalyseur des changements économiques et sociaux ${ }^{69}$. Cette analyse justifie son pouvoir autoritaire car le bon gouvernement est celui qui assure et encadre le développement économique.

Cette pensée trouve là encore un terreau favorable en Espagne dans la pensée ilustrada, très sensible aux idées physiocratiques puis au libéralisme. L'État est perçu comme le garant des libertés économiques, mais aussi comme l'aiguillon de l'activité économique, le responsable du fomento. Cependant, le but ultime de la recherche de l'enrichissement du pays est le développement de la puissance de l'État ${ }^{70}$. Ces analyses expliquent le large succès de l'économie politique, considérée comme la «médecine du corps mystique de l'État $»^{71}$.

Les réformes de fond entreprises sous Joseph Bonaparte vont dans cette direction, mais elles ne purent être réellement appliquées. Il en fut de même pour une bonne part des initiatives du Trienio. La réflexion théorique qui marque la dernière phase du règne de Ferdinand VII, insiste sur la place centrale que doit occuper l'économie. Si des libéraux en exil en furent les acteurs (José Canga Argüelles, Alvaro Flórez Estrada), les anciens afrancesados occupèrent là une place de premier plan. Ces motivations sont celles qui prévalent dans les travaux de Pedro Sainz de Andino avec l'adoption de son Code du commerce en 1829 et la fondation de la Bourse de Madrid (10 décembre 1831) dont il est l'inspirateur. On trouve même des accents industrialisateurs chez Burgos quand il vante l'introduction des machines: «en augmentant la production, on crée de nouvelles ressources destinées à en produire de nouvelles dans une progression infinie $»^{72}$.

Cette pensée se nourrit très tôt, dès la fin des années 1810 , de certains éléments du saint-simonisme, confirmant ainsi les interactions entre bonapartisme et saintsimonisme que le Second Empire concrétisera. Le passionnant complot de 1819 révélé récemment par Claude Morange voit les conspirateurs, dans une précoce union entre libéraux modérés et afrancesados, refuser le suffrage universel et le réserver aux "producteurs et industriels $»^{73}$. Quinze ans plus tard, Lista évoque «la classe industrielle " pour désigner l'ensemble des producteurs ${ }^{74}$. Le primat saint-simonien de l'économie sur la politique renforce une tradition antidémocratique qui justifie l'existence d'un État autoritaire (qu'il soit, avec certes des degrés, absolutiste réformateur, bonapartiste ou libéral modéré) dominé par une administration toute puissante assurant un développement économique qui est seul capable d'asseoir un changement politique durable. La force de cette logique se retrouve même chez des hommes qui ne sont pas des spécialistes de l'administration. Lors de la discussion de la fin de l'année 1830 sur l'opportunité de créer un ministère de l'Intérieur, Zambrano, ministre de la Guerre, affirmait : " le ministère de l'Intérieur est une création des temps modernes et des progrès administratifs : c'est un effet des progrès industriels et mercantiles $»^{75}$.

Le primat de l'économie repose sur une approche "scientifique" du fonctionnement des sociétés. Les lumières de "l'expérience et de la raison" amènent à rechercher et respecter les règles $d u$ "bon gouvernement» consignées en particulier dans le droit administratif. L'exclusion du plus grand nombre du débat et de la participation à la vie 
politique prôné dès la fin du XVIIIe siècle par les ilustrados trouve alors avec les modérés dans les années 1840 une justification renouvelée, dans laquelle les afrancesados et certains aspects de l'expérience napoléonienne jouèrent un rôle essentiel.

Paradoxes et limites de l'influence napoléonienne

L'exemple de la France modelée par Bonaparte a marqué indéniablement un milieu et une mouvance politique : les élites sociales acquises au modérantisme. Qu'en est-il du reste de la société espagnole ? Les milieux populaires semblent totalement hermétiques aux charmes de la légende napoléonienne, campant sur le rejet et la francophobie. La mouvance libérale révolutionnaire (de Cadix aux proto-républicains des années 1830, en passant par les exaltés du Trienio liberal et les progressistes du règne d'Isabelle II) écarte le modèle français pour son autoritarisme. Toutefois, le rejet n'est pas sans ambiguïté : l'aventure d'Espartero a indéniablement des accents bonapartistes.

Les contre-révolutionnaires sont bien sûr dans une opposition totale, pourtant, c'est paradoxalement chez Ferdinand VII que l'on peut déceler une influence napoléonienne que l'on n'attendait pas. Il fut tout d'abord poussé dans la dernière décennie de son règne à accepter une politique réformatrice héritée des méthodes du despotisme éclairé et matinée de libéralisme qui avait indéniablement quelques accents napoléoniens. Un autre domaine porte la marque de l'empereur : celui de l'art et de son utilisation à des fins de propagande. Ferdinand VII connaissait en effet de près Napoléon et la France napoléonienne par l'exil qu'il dut subir durant presque cinq ans à Valençay, pendant la guerre d'Indépendance. Cet épisode est très mal connu, mais c'est probablement là qu'il a compris que le chef de l'État, le grand homme, devait désormais se mettre en représentation de manière diversifiée et massive ${ }^{76}$. Aucun souverain espagnol ne fut autant représenté que Ferdinand VII. Les tableaux à sa gloire sont très nombreux, les plus importants ont été réalisés par des artistes néoclassiques (Francisco Lacoma, Vicente López...) qui ont séjourné longuement à Paris où certains furent élèves de David, avant d'être désignés peintres de la Cour par Ferdinand ${ }^{77}$. On est frappé en visitant le Musée Romantique de Madrid de la grande diversité des supports à l'effigie du roi : vaisselle, éventails, montres, plateaux, estampes... Diversité aussi dans les types de représentation du souverain : tantôt selon des canons classiques (en majesté ou à cheval), tantôt en simple costume bourgeois. Comme l'empereur, Ferdinand est très souvent représenté dans les moments forts de son activité politique. À défaut de gloire militaire, il s'agit surtout des deux moments durant lesquels il retrouva son trône de monarque absolu, au printemps 1814 et à l'automne 1823, moments d'intense propagande, largement mythifiés par la suite.

Ferdinand VII comprit aussi que le prestige du prince se mesure également à l'aune de ses activités de mécène et de collectionneur. Les efforts financiers consentis dans ce domaine sont révélateurs au regard de la situation calamiteuse du Trésor Royal. Une telle politique apparait dans l'ameublement et la décoration du Palais Royal de Madrid qui sont pour une part importante le fruit des achats de Ferdinand VII (par exemple de superbes horloges), mais surtout dans la création du musée du Prado en 1819. Même si son inauguration ne semble pas avoir donné lieu à des manifestations d'ampleur, la création d'un musée constitué des collections royales et placé sous la dépendance de la Maison Royale est présenté comme le résultat d'une volonté personnelle du roi ${ }^{78}$. En appuyant la création de ce musée, qui avait été rejetée par le Conseil de Castille à plusieurs reprises depuis 1814, Ferdinand s'inscrit dans le prolongement des initiatives 
de Joseph Bonaparte qui avait joué un rôle de précurseur en 1809 avec son projet de musée joséphin ${ }^{79}$.

Cet exemple montre que la force de l'expérience napoléonienne en fait une référence qui laisse des traces même quand elle est rejetée. Elle n'est toutefois jamais revendiquée sans réserve après 1814. Les afrancesados puis les libéraux modérés ont retenu surtout $\mathrm{du}$ modèle politique napoléonien son caractère autoritaire et rationalisateur, son souci du développement économique dans le but d'accroître la puissance de l'État. Ils l'ont débarrassé de tout romantisme et de toute mystique populaire. En cela, l'exemple français est assimilé pour donner des outils à une voie empruntée dès avant le Consulat: celle qui conduit de la ilustración aux modérés des années 1840 et qui cherche à faire sauter les verrous de la société d'Ancien Régime sans intervention populaire. Dans ce but, un rôle central est dévolu à l'État. L'influence napoléonienne en Espagne s'insère ainsi dans une histoire nationale que l'on peut elle même inscrire dans une évolution européenne plus large, y compris dans le domaine qui apparaît comme le plus "français", celui du droit ${ }^{80}$. L'application de ce modèle politique s'est cependant heurtée à deux réalités essentielles : la mobilisation populaire et la pauvreté de l'État. L'irruption du peuple entamée par la Guerre d'Indépendance s'avère nécessaire jusqu'aux années 1840 pour vaincre une contre-révolution puissante. Ce danger s'éloignant, la recherche de la dépolitisation du pays par les modérés devient un mirage. Ceci est d'autant plus utopique que les profits que le pays pouvait retirer d'un tel modèle ne sont pas au rendez-vous : contrairement à la France, l'État est dans l'impossibilité d'assurer le moindre investissement public et n'a même pas les moyens de garantir l'ordre intérieur ou de contrôler les frontières ${ }^{81}$. Le bel échafaudage intellectuel des théoriciens de l'administration ne tient pas devant la crise économique et l'égoïsme des possédants anciens et nouveaux qui empêchent toute augmentation notable du produit des impôts. Au fond, les élites au pouvoir n'assument pas toutes les conséquences, en particulier fiscales, du modèle qu'elles avaient contribué à élaborer ${ }^{82}$. Les frustrations n'en furent que plus intenses.

\section{NOTES}

1.Ricardo Garcia Carcel, La leyenda negra. Historia y opinión, Madrid, 1998.

2.Jean-René Aymes, La déportation sous le Premier Empire. Les Espagnols en France

(1808-1814), Paris, 1983. Rafael Sanchez Mantero, Liberales en el exilio. La emigración política en Francia en la crisis del antiguo Régiment, Madrid, 1975.

3. "Exposición dirigida al señor D. Fernando VII desde Paris en 24 de enero de 1826 ", dans Javier de Burgos, Anales del reinado de Doña Isabel II, t. I, Madrid, 1850, p. 64.

4.Alberto Gil Novales, « La dualidad napoleónica en España », dans Les Espagnols et Napoléon, Actes du colloque international d'Aix-en-Provence, 13-14-15 octobre 1983, Aix-en-Provence, Université de Provence, 1984, p. 7-24.

5.Cité par A. Gil Novales, op. cit., p. 11. Outre ce colloque d'Aix-en Provence, je me contenterais dans une bibliographie foisonnante d'évoquer : Jean-René Aymes (dir.), L'image de la France en Espagne (1808-1850), Paris, 1997. 
6.Jean-René Aymes, « Deux historiens libéraux espagnols face à Napoléon : le comte de Toreno et Francisco Martínez de la Rosa » dans Les Espagnols et Napoléon, op. cit., p. 101-119.

7.Irene Castells, La utopía insurreccional del liberalismo. Torrijos y las conspiraciones liberales de la década ominosa, Barcelona, 1989, p. 134.

8.A. Gil Novales, op. cit., pp. 15-16.

9.Irene Castells, « La opinión liberal ante la invasión francesa: Cataluña (1823) » dans Juan Francisco Fuentes, Lluís Roura, Sociabilidad y liberalismo en la España del siglo XIX ; Homenaje a Alberto Gil Novales, Lleida, 2001, pp. 150-152. Je remercie Irene Castells pour les informations et les précisions qu'elle m'a apportéés sur cette question.

10.Kobo Seigan, « L'influence de la mémoire de la révolution française et de l'empire napoléonien dans l'opinion publique française face à la guerre d'Espagne de 1823 », A.H.R.F., nº1, 2004, pp. 159-181.

11.Propos de Manuel María Guttierrez, dans un ouvrage de 1841. Cité par A. Gil Novales, op. cit., p. 16.

12.Miguel Artola, Los afrancesados, Madrid, 1943 (édition utilisée : Madrid, 1989). Hans Juretschke, Los afrancesados en la Guerra de la Independencia, Madrid, 1962. Jaime Mercader Riba, José Bonaparte, rey de España, historia externa del reinado, Madrid, 1971 et Ibid, estructura del Estado bonapartista, Madrid, 1983. Manuel Moreno Alonso, La generación española de 1808, Madrid, 1989. Luis Barbastro Gil, Los afrancesados, primera emigración política del siglo XIX español (1813-1820), Madrid, 1993. Juan Lopez Tabar, Los famosos traidores. Los afrancesados durante la crisis del Antiguo Régimen (1808-1833), Madrid, 2001. Ce dernier ouvrage est devenu incontournable, car outre une synthèse récente des travaux réalisés, il intégre le phénomène afrancesado dans la durée, dans le processus global de crise et fin de l'Ancien Régime.

13.Décret du 30 mai 1814. Le texte est reproduit dans : M. Artola, op. cit., p. 247-248. 14.Ordonnance du 26 avril 1820. M. Artola, op. cit., p. 234.

15.Agustín Martinez de las Heras, "Los periódicos afrancesados, absolutistas y ultras vistos desde el universal (1820-1823)" dans J. F. Fuentes, L. Roura, op. cit., pp. 103-116. 16. Hans Juretschke, op. cit., p. 563.

17.J. López Tabar, Los famosos traidores (...), pp. 262-285.

18.J'ai développé les étapes de ces négociations dans : Jean-Philippe Luis, « le difficile et discret retour des afrancesados (1816-1834) » dans Rose Duroux, L'émigration. Le retour, Clermont-Ferrand, 1999, p. 335.

19.Archivo Histórico Nacional, Consejos, libro 989, pp. 840-858 pour l’intégralité de la consulta.

20.Plus de détails dans l'application de l'amnistie dans : J.-Ph. Luis, op. cit., pp. 336-337. 21.Article Afrancesados dans Javier Fernandez Sebastian, Juan Francisco Fuentes (dir.), Diccionario político y social del siglo XIX español, Madrid, 2002, p. 79.

22.Antoine Casanova, «L'influence juridique française en Italie au XIXe siècle », Revue d'Histoire des Facultés de droit et de la science juridique, nº 15, 1994.

23.Pour ce qui suit sur cette question : Jean-Philippe Luis, L'utopie réactionnaire Épuration et modernisation de l'État dans l'Espagne de la fin de l'Ancien Régime (1823-1834), Madrid, 2002.

24.Mémoire daté du 12 juillet 1826 présenté au comte de Villéle, Archivo de Palacio, Papeles reservados de Fernando VII, t. 70, $n^{\circ} 8$. 
25.Antonio Elorza, La ideologia liberal en la ilustración española, Madrid, 1970. A. Romano (dir.), Il modelo costituzionale inglese e la sua recezione nell'area mediterranea tra la fine del 700 e la primametá dell'800, Milano, 1998.

26.Juan Alfonso Santamaria Pastor, Sobre la genesis del derecho administrativo español en el siglo XIX. 1812-1845, Sevilla, 1973, p. 29.

27.Joaquín Varela Suanzes, « El pensamiento constitucional español en el exilio : el abandono del modelo doceanista (1823-1833) », Revista de Estudios Políticos, $\mathrm{n}^{\circ}$ 88, abriljunio 1995, pp. 63-90.

28. Alejandro Nieto, Los primeros pasos del estado constitucional, Barcelona, 1996, p. 89. 29.Je renvoie ici aux travaux des juristes : Jean-Louis Mestre, Introduction historique au droit administratif français, Paris, 1985 ; François Burdeau, Histoire du droit administratif. De la Révolution au début des années 1970, Paris, 1995.

30.Mariano Baena del Alcazar, Los estudios sobre administración en la España del siglo XVIII, Madrid, 1968, pp. 56-70.

31.J.-Ph. Luis, L'utopie réactionnaire (...), op. cit., pp. 198-203.

32.J. de Burgos, op. cit., p. 64.

33.Jesús Burgueño, Geografía política de la España constitucional. La division provincial, Madrid, 1996.

34.De nombreux éléments dans l'ouvrage déjà ancien : Ascension Fornies Baigorri, La vida comercial española. 1829-1885, instituciones, doctrina y legislación mercantil, Zaragoza, 1968.

35.Jean-Louis Mestre, « Administration, justice et droit administratif », A.H.R.F., $\mathrm{n}^{\circ}$ 328, avril/juin 2002, p. 73.

36. Compendio de los principios de Administración, traducida al castellano por D. J. M. Saavedra, Madrid, 1834.

37. Cité par A. Nieto, op. cit., pp. 84-85.

38.Ibid., pp. 86-87.

39.Eduardo Garcia de Enterria, « Alejandro Oliván y los orígenes de la Administración española contemporánea », dans La administración española. Estudios de ciencia administrativa, Madrid, 1972, p. 31.

40.F. Burdeau, op. cit., pp. 74-75.

41.J. A. Santamaria Pastor, op. cit., pp. 143-152.

42.Napoléon et la pensée de son temps : une histoire intellectuelle singulière, Paris, 2001.

43.L. Barbastro Gil, op. cit., pp. 137-138.

44.Jovellanos, la grande conscience libérale et patriote du début de la Guerre d'Indépendance, associait dès les années 1780 la garantie de la liberté à celle de l'ordre social. Sur la méfiance à l'égard du peuple : Juan Francisco Fuentes, « El concepto de pueblo en el primer liberalismo español », Trienio, 1988, n 12, pp.176-209.

45.Juan Lopez Tabar, « Los afrancesados y la voluntad de reconciliación », Trienio, $\mathrm{n}^{\circ} 29$, mayo 1997, p. 79.

46.Ibid., pp. 73-76.

47.J. López Tabar, Los famosos traidores (...), op. cit., pp.318-339.

48.Le rapprochement n'est pas immédiat et s'avère conflictuel. Il ne suit pas immédiatement la mort du roi car les afrancesados de premier plan (Burgos, Lista ...) soutenaient le projet de Cea Bermúdez de réformisme dans le cadre d'une monarchie absolue, d'où les violentes attaques venant des libéraux modérés qu'ils durent subir en 1833-1834. Antonio Elorza, La modernización política de España, Madrid, 1990. Ibid., pp.

233-235. 
49.Article Moderado dans : Diccionario político y social del siglo XIX español, op. cit., p. 449.

50.A. Casanova, op. cit., p. 114.

51.Ibid., p. 120.

52.Article Clase media dans : Diccionario político y social del siglo XIX español, op. cit., p. 161.

53. Cité par A. Casanova, op. cit., p. 116.

54.Ibid., pp. 120 et 124 .

55. Cité dans A. Elorza, La modernización política ..., op. cit., p. 220.

56.Ibid., p. 222.

57.Ibid., p. 225.

58.Pierre Rosanvallon, Le sacre du citoyen. Histoire du suffrage universel en France, Paris, 1992, p. 326.

59.Voir l'excellent article de Claude Morange, « opinión pública: cara y cruz del concepto en el primer liberalismo español », dans J. F. Fuentes, L. Roura (ed.), op.cit., pp. 117-145.

60.A. Elorza, La modernización política..., op. cit., p. 219.

61.Annie Jourdan, Napoléon, héros, imperator, mécène, Paris, 1998, pp. 70-81.

62.P. Rosanvallon, op. cit., pp. 266-267.

63.Santos M. Corona Gonzalez, « Las leyes fundamentales del Antiguo régimen (notas sobre la Constitución histórica española) » Anuario de Historia del Derecho español, 65 (1995), pp. 127-218. Fernando Baras Escola, El reformismo político de Jovellanos. Nobleza y poder en la España del siglo XVIII, Zaragoza, 1993.

64.Article Soberanía dans ibid., p. 649.

65.José María Portillo valdes, Revolución de nación: orígenes de la cultura constitucional en España 1780-1812, Madrid, 2001.

66.Cité dans A. Elorza, La modernización política..., op. cit, p. 225.

67.Voir l'article Nación dans Diccionario político y social del siglo XIX español, op. cit., pp. 472-473.

68. María Cruz Romeo, « Lenguaje y política del nuevo liberalismo: moderados y progresistas, 1834-1845 », Ayer, n² 29, 1998, p. 43.

69.A. Casanova, op. cit., pp. 197-201.

70.Josep Fontana, « "El alimento del Estado”. Política y Hacienda en el "despotismo ilustrado" ", Hacienda Pública Española, n 108-109, 1987, pp. 157-168.

71.Propos tenus par Alcalá Galiano en 1813, cité dans l'article Economía política dans : Diccionario político y social del siglo XIX español, op. cit., p. 251.

72.J. de Burgos, op. cit., p. 66.

73. Claude Morange, « El programa político de la conspiración de 1819 », Trienio, n³9, mai 2002, p. 38.

74.A. Elorza, La modernización política ..., op. cit., p. 233.

75.Mémoire de Zambrano au Roi, dans Pedro Sainz de Andino. Escritos, t. III, (Documentos del reinado de Fernando VII, V), Pamplona, 1968, p. 268.

76.Je renvoie bien sûr à l'indispensable ouvrage d'A. Jourdan, op. cit.

77.Eliseo Trenc Ballester, «Los pintores neoclásicos españoles en París », dans Jacques Maurice, Marie-Claire Zimmermann (comp.), Actas del XXVIII congreso de la sociedad de hispanistas franceses, Paris, 1998, pp. 199-206.

78.Pierre Géal, Recherches sur la naissance des musées d'art en Espagne de Charles III à Isabelle II, Thèse de doctorat, université de Paris IV, 1997, pp. 158-159.

79.Ibid., chapitre IV. 
80.Luigi Lacché, « L'Europe et la révolution du droit : brèves réflexions », A.H.R.F., 2002, $\mathrm{n}^{\circ} 2$, pp. 153-169.

81.Albert Broder, Histoire économique de l'Espagne contemporaine, Paris, 1998, pp. 42-45.

82.Une abondante bibliographie récente existe sur l'État libéral. Voir en particulier le numéro spécial : Historia contemporánea, n 17, 1998.

\section{RÉSUMÉS}

Se réclamer de l'influence napoléonienne est impossible dans l'Espagne des lendemains de la Guerre d'Indépendance. Pourtant, l'évolution de la Révolution libérale qui vit progressivement l'emporter un libéralisme oligarchique conservateur valorisa de plus en plus ouvertement un modèle d'État qui est celui de la France post-napoléonienne, centralisateur, uniformisateur et autoritaire. L'emprunt fait au modèle napoléonien porte sur l'art de la propagande, et surtout sur le modèle juridico- administratif. L'État libéral qui se met progressivement en place aux lendemains de la Guerre d'Indépendance porte la marque certaine de l'influence française confirmée par le rôle de l'exemple français dans la genèse du droit administratif espagnol- tout en s'insérant dans le même temps dans la tradition du réformisme du despotisme éclairé qui est la version espagnole d'un vaste mouvement européen de transformation des sociétés d'Ancien Régime. Par ailleurs, l'emprunt à la France n'est que partiel : le modèle est dépouillé de tout romantisme et de toute mystique populaire. Il exclut totalement les masses, puis dans une large mesure les classes moyennes pour limiter l'exercice du pouvoir à une étroite élite. Il fait ensuite l'impasse sur les moyens financiers indispensables à l'existence d'un État fort.

\section{The influence of the Napoleonic Model in Spain (1814-1845)}

To claim allegiance to the Napoleonic heritage was impossible in Spain after the war of independence. Yet the evolution of the liberal revolution which saw the gradual triumph of a conservative oligarchic liberalism, tended openly to validate a model of state which was that of post-Napoleonic France, featuring centralization, uniformity and authority. Adoption of the Napoleonic model comprised the art of propaganda and especially the legal administrative model. The liberal state which gradually emerged after the war of independence bore the stamp of French influence - confirmed by the role of the French example in the development of Spanish administrative law - while simultaneously espousing the reformist tradition of enlightened despotism, the Spanish version of the great European trend to transform the old regime societies. Moreover, the debt to France is partial, the model being robbed of its romanticism and popular mystique. It totally excludes the masses, and also to a large extent the middle classes, confining the exercise of power to a narrow élite, thus running up against a sorry lack of funding necessary to a strong state.

\section{INDEX}

Mots-clés : Espagne, administration, propagande, Napoléon, afrancesados, modérés, Ferdinand VII 
AUTEUR

JEAN-PHILIPPE LUIS

Université Blaise Pascal - Clermont-Ferrand 